\section{A digital examination of medicine}

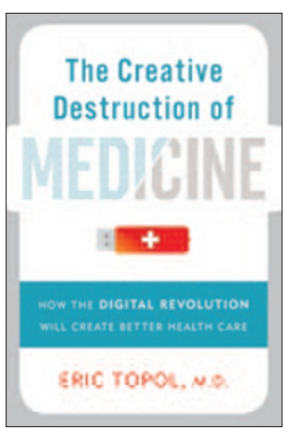

\section{The Creative Destruction of \\ Medicine: How the Digital \\ Revolution Will Create Better Healthcare}

\section{Eric Topol}

320 pp., hardcover, $\$ 56.99$

ISBN: 0465025501

\section{Reviewed by Hadrian A L Green}

Basic Books, 2012

As a professor of cardiology, Eric Topol is well positioned to recalibrate beliefs about the current performance of the healthcare system. Indeed, the successive '-omic' revolutions are revealing that common medical presentations are actually families of disease with distinct underlying biologies that each imply a very different prognosis and treatment. Yet the routines of the ward today depart little from those at the beginning of the last century, continuing to depend on clinical symptoms and signs documented in handwritten notes.

Deriving inspiration from the economist Joseph Schumpeter, Topol proposes nothing less than the 'creative destruction' of medicine as it is currently practiced, replacing it with a brave new world in which interconnected technologies dramatically improve patient outcomes. The thesis at the center of his book is that medicine has resisted the 'digital revolution' and its successive waves of technological convergence between diverse and hitherto unrelated fields, including the Internet, biotech, the development of smartphones and data mining. Topol makes the case that this convergence has given us the capability to interrogate a wide variety of data from disparate sources-such as demography, biosensors, imaging and genomics. He argues that, by utilizing these existing technologies, we can surpass any given doctor's ability to recognize the relationship between diseases and various biomarkers and understand in a much more individualized way the likely impact of current treatments on specific patients. Topol's analysis is one manifestation of the growing recognition that as the world has moved from being 'information poor' to 'information rich', the bottleneck has moved from generating information to collating and mining vast clinical datasets.

Under the aegis of this central idea, Topol offers wide-ranging examples, such as the use of CYP2C19 genotyping to typify clopidogrel metabolism, to show a proof of principle and highlight the implications of his thesis for morbidity and healthcare costs. Indeed, so rapid is the flow of ideas and examples that readers may struggle to distinguish those ready for application in the clinic from those at a more experimental stage.

Topol rightly identifies the fragmentation of medical information and the lack of agreed data standards as limits to the consolidation of data garnered during the innumerable natural experiments of everyday patient-

Hadrian A.L. Green is in Emerging Markets Research and Development at GlaxoSmithKline, Brentford, UK. doctor interactions, restricting the sophistication of resulting insights. Electronic health record systems are another key technology visited in the book, and the example of the association of COX2 inhibitors with heart attacks discovered using the Kaiser Permanente database amply shows what sort of results can be delivered with a properly configured system. Yet for all this promise, such systems are extremely difficult to implement for use by doctors with current clinical information; future expansions in clinical datasets and the attendant complexity of identifying disease associations will present further problems. The recent abandonment of a $£ 12$.4 billion initiative to implement nationwide electronic medical records in the UK provides a timely reminder of the challenges faced, even by a government with an effective monopoly on healthcare. Because it is not clear that the centralized (potentially Orwellian) system required to deliver Topol's vision can feasibly effect the revolution he calls for, we must wonder whether his proposal is in fact a deus ex machina. Instead, as most of the technologies advocated have commercial applications, perhaps an approach premised upon Adam Smith's "invisible hand of the market" would have more appeal to his readership. By blaming previous failures on stakeholder resistance, Topol instead risks becoming partisan.

Topol proposes that we overcome the limitations of current diagnostics by massively expanding the number of biomarkers considered. Both the potential success and the potential difficulties inherent to this strategy are illustrated by the example of Oncotype DX, one of the few successful multi-marker diagnostics on the market, which is thus a surprising omission from the book. The test developed by Oncotype DX computes a recurrence score to identify the patients most likely to benefit from chemotherapy for their breast cancer. The high cost of developing this technology, which uses only 21 relatively well-understood markers, indicates the likely investment needed to incorporate the hundreds (or even thousands) of data points that Topol would want to analyze, notwithstanding economy gained via Moore's Law. Before allowing such information to dictate treatment guidelines, it would also be necessary to evaluate each new disease association (for example, by Bradford-Hill's criteria). Many readers will suspect that we may need to settle for incremental change and that wholesale changes in patient outcomes from -omics of the kind described here still remain just over the horizon.

Topol is in a rare position, having been a witness to both the beginnings of the personal computing revolution and the first biotech medicines. The highly personal nature of his account results, however, in a US-centric view that may vex international readers. Given the leading position of US biotech, this is not to the detriment of the book, but the idiosyncrasies of the US healthcare system limit the general applicability of some of its insights.

Overall, the book is an enjoyable, high-level review of the current state of the field, intended for a general audience but referenced for those inclined to read more deeply. With its rich discussion of science and technology and companies specific to the last couple of years, this book certainly has contemporary relevance. It will be fascinating to see whether future healthcare technologies converge as Topol supposes. Sometimes you have to wait a long time for a revolution.

\section{COMPETING FINANCIAL INTERESTS}

The author declares competing financial interests: details accompany the full-text HTML version of the paper at http://www.nature.com/naturegenetics/. 